\title{
一种新型酰胺功能化的共价有机框架用于选择性染料吸附
}

\author{
方婧＼cjkstart赵文娟＼cjkstart张明浩＼cjkstart方千荣* \\ (吉林大学化学学院 无机合成与制备化学国家重点实验室 长春 130012)
}

\begin{abstract}
摘要 共价有机框架(covalent organic framework, $\mathrm{COF}$ ) 是一种由轻质元素 $(\mathrm{C} 、 \mathrm{H} 、 \mathrm{O}$ 和 $\mathrm{N}$ ) 以共价键的形式连接组成的结 晶多孔聚合物, 由于其具有规则的孔道、可修饰的骨架以及良好的稳定性而被广泛应用于不同的领域. 尤其是将含氮 的功能基团连接到 COF 的骨架中, 可以为其吸附特定的染料提供丰富的活性位点. 基于此, 本工作成功制备了一种酰 胺功能化的二维共价有机框架材料(JUC-578), 通过一系列的表征证明了该材料具有高的结晶度、均一规整的形貌以及 开放的一维介孔孔道. 更重要的是, 发现 JUC-578 可以选择性地吸附阳离子染料, 并且可以多次循环利用. 这主要归因 于骨架中的氮作为电子给体与缺电子的染料之间的静电作用以及其他弱相互作用 (氢键、偶联作用等). 与此同时, JUC-578 高的结晶度和有序的孔道也是实现可逆吸附染料非常重要的因素.

关键词 多孔材料; 共价有机框架; 晶体结构; 酰胺功能化; 染料吸附
\end{abstract}

\section{A Novel Amide-functionalized Covalent Organic Framework for Selective Dye Adsorption}

\author{
Jing Fang Wenjuan Zhao Minghao Zhang Qianrong Fang* \\ (State Key Laboratory of Inorganic Synthesis and Preparative Chemistry, School of Chemistry, Jilin University, \\ Changchun 130012, China)
}

\begin{abstract}
Organic dyes are widely applied for cloth dyeing process, paper printing, cosmetics, and food processing industries. The toxicity of industrial dyes poses serious threats to water and human health. Thus, several methods such as adsorption, degradation, and photocatalytic oxidation or reduction have been proposed to remove dyes from water. Among them, with low cost and high adsorption efficiency, porous materials become the most common materials to remove organic dyes from aqueous solutions. Notably, covalent organic frameworks (COFs) are a kind of novel crystalline porous polymers with regular pores, modifiable frameworks, high specific areas and excellent stability. Because of their outstanding properties, COFs have been used in many fields, such as gas adsorption and separation, heterogeneous catalysis, semiconductors and sensors. Especially the introduction of the nitrogen-containing functional groups to the COF skeletons provides abundant active sites to adsorb specific dyes in the field of dye adsorption. Inspired by this, a novel amide-functionalized two-dimensional COF (termed as JUC-578) was successfully prepared through Schiff-based condensation reaction of 4,4'-diaminobenzanilide (DABA) and 1,3,5-benzenetricarboxaldehyde (BT). A series of characterizations proved that JUC-578 has high crystallinity, uniform morphology and opening one-dimensional mesoporous pores. Especially the morphology of JUC-578, which was characterized by scanning electron microscope (SEM) and transmission electron microscope (TEM), showed a clear spherical structure with uniform morphology distribution and smooth surface. Moreover, we studied the reversible adsorption of JUC-578 on dyes from aqueous solutions by UV-Vis spectrophotometry. The results show that JUC-578 can selectively adsorbed cationic dyes and the structure remained stable after cycling, which was attributed to the electrostatic interaction between the electron donor nitrogen in the skeleton and the electron-deficient dyes as well as other weak interactions (hydrogen bonding, coupling, etc.). Meanwhile, the crystallinity and ordered pores of JUC-578 are also important factors for reversible dye adsorption. These results indicate that COFs as functionalized porous materials have great potential in dealing with environmental problems.

Keywords porous material; covalent organic framework; crystal structure; amide-functionalized; dye adsorption
\end{abstract}

\section{1 引言}

有机染料作为一种重要的工业原料, 已被广泛应用 于丝绸、棉、亚麻、涤纶等纤维的染色和造纸工业. 但 是由于一些染料具有毒性、致癌性以及在光和氧化等环 境中稳定的性质, 使其在水中难以降解, 从而造成了严
重的水污染，因此寻找合适的方式来除去水中的有机染 料是叒待解决的问题 ${ }^{1-2]}$. 目前, 去除水中染料的方法有 吸附、降解、光催化氧化或还原等. 在这些方法中, 由 于多孔材料具有吸附成本低、效率高等优势, 被认为是 从水溶液中去除有机染料最理想的材料 ${ }^{[3]}$. 已经有多种

* E-mail: qrfang@jlu.edu.cn

Received October 14, 2020; published November 23, 2020.

Project supported by the National Natural Science Foundation of China (No. 21571079).

项目受国家自然科学基金(No. 21571079)资助. 
多孔材料被用来去除染料, 比如活性炭 ${ }^{[4]}$ 、石墨烯 ${ }^{[5]}$ 、有 机二氧化硅 ${ }^{[6]}$ 和金属有机框架(MOF) ${ }^{[7]}$, 尤其 MOF 展现 出快速的吸附动力学和高饱和容量, 证明了晶态多孔材 料在吸附剂设计中的实用性 ${ }^{[8-9]}$. 然而, 由于相对不稳定 的金属有机配位键, MOF 的低稳定性阻碍了染料的有效 去除、材料的回收和再利用 ${ }^{[10]}$.

共价有机框架 $(\mathrm{COF})$ 是一种由轻质元素 $(\mathrm{C} 、 \mathrm{H} 、 \mathrm{O}$ 和 N) 以共价键的形式连接组成的结晶多孔聚合物 ${ }^{[11]}$, 由于其具有低的骨架密度、高的比表面积以及丰富的孔 道而被广泛应用于气体吸附与分离 ${ }^{[12]}$ 、多相催化 ${ }^{[13-14]}$ 、 半导体 ${ }^{[15-16]}$ 、传感器 ${ }^{[17]}$ 、能源存储 ${ }^{[18]}$ 等领域. 与其他多 孔材料相比, COF 具有规则的孔道、可修饰的骨架、高 的比表面积以及良好的稳定性, 能够有效地去除溶液中 有害的染料分子. 尤其是将含氮的功能基团连接到 COF 的骨架中, 为 COF 吸附特定的染料提供了丰富的 活性位点 ${ }^{[19]}$. 例如, Zhu 等 ${ }^{[20]}$ 制备得到的共价三嗪框架 (CTF)中存在丰富的氮原子, 可作为碱活性位点与阴离 子染料的自由电子相互作用, 而对于阳离子染料的吸 附, 涉及静电和分散相互作用这两种平行的吸附机制. 在其他多孔材料中同样证明了含氮功能基团对染料的 特殊吸附作用, 例如 $\mathrm{Su}$ 等 ${ }^{[9]}$ 报道的含有四唑的中性 MOF 暴露了大量的氮活性位点, 利用氮上的孤电子对 具有给电子体的特性, 供体-受体(D-A)相互作用来选择 性地吸附缺电子的染料. 另外, Chen 等 ${ }^{[21]}$ 报道了酰胺功 能化的碳纳米球, 通过在表面引入酰胺基团增强了碳纳 米球和染料分子之间的氢键和偶联相互作用, 从而为染 料的吸附提供了额外的吸附位点. 因此, 设计具有含氮 功能基团的 COF 材料用于染料的选择性吸附是一种可 行的方案.

基于上述考虑, 我们选取 4,4'-二氨基苯甲酰苯胺 (DABA) 和均苯三甲醛(BT)通过溶剂热的方法制备得到 了一种新型酰胺功能化的二维 COF (JUC-578), 如图式 1 所示. JUC-578 具有高的结晶度、均一规整的形貌以

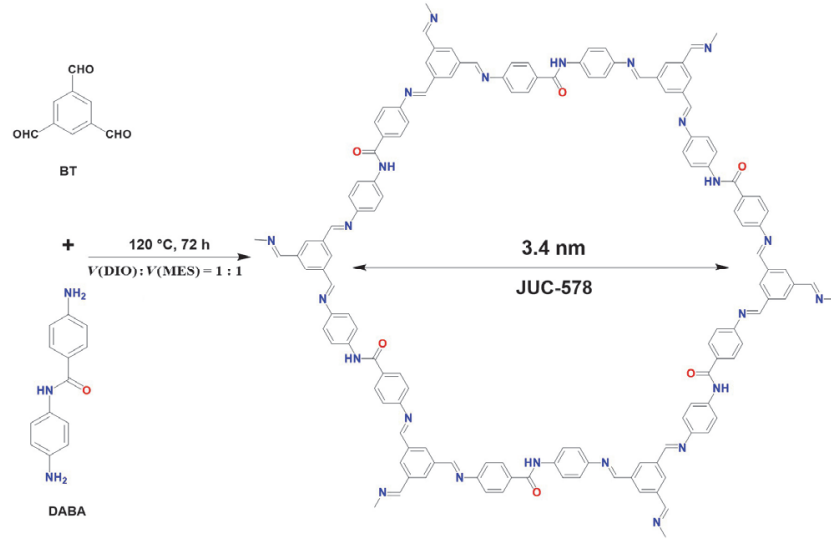

图式 1 JUC-578 的合成

Scheme 1 Synthesis of JUC-578
及一维介孔孔道，这有利于染料分子在孔道中的扩散以 及与活性位点的结合. 此外, 通过扫描电子显微镜 (SEM)和透射电子显微镜(TEM)表征了 JUC-578 的形貌, 可以很清晰地看到形态分布均匀且表面较为光滑的球 状结构. 更重要的是, JUC-578 可以在水溶液中选择性 地吸附阳离子染料，这可能主要归因于骨架中酰胺官能 团与亚胺键提供了丰富的氮活性位点, 与缺电子的染料 之间存在强的静电相互作用.

\section{2 结果与讨论}

\subsection{JUC-578 的表征}

基于上述设计思路，我们成功制备了 JUC-578，并 采用粉末 $X$ 射线衍射(PXRD)、SEM、TEM、 $\mathrm{N}_{2}$ 吸附、 红外光谱图(FT-IR)等多种方法表征了材料的晶体结构.

通过 SEM 用来表征 JUC-578 的形貌和颗粒大小, 如图 $1 \mathrm{a}$ 和 $1 \mathrm{~b}$ 所示, 可以很清晰地看到形态分布均匀的 圆球状结构, 且每一颗圆球独立规整的均匀排布, 粒径 约为 $1 \mu \mathrm{m}$. 通过 TEM(图 $1 \mathrm{c}$ 和 $\mathrm{d}$ )更能清楚地看到圆球 表面均一光滑无残缺. 为了进一步确定材料的结构, 我 们测定了配体以及 JUC-578 的 FT-IR. FT-IR 清楚地表明 JUC-578 在 $1685 \mathrm{~cm}^{-1}$ 和 $1602 \mathrm{~cm}^{-1}$ 处出现了 $\mathrm{C}=\mathrm{O}$ 和 $\mathrm{C}=\mathrm{N}$ 伸缩振动的新峰, 而起始配体 $\mathrm{DABA}$ 的 $\mathrm{N}-\mathrm{H}$ 伸 缩振动峰 $\left(3200 \sim 3400 \mathrm{~cm}^{-1}\right)$ 和 $\mathrm{BT}$ 的 $\mathrm{C}=\mathrm{O}$ 伸缩振动峰 (1698 $\mathrm{cm}^{-1}$ )的消失, 证实了醛基和氨基的完全转化(图 2a). 根据固态 ${ }^{13} \mathrm{C}(\mathrm{CP} / \mathrm{MAS}) \mathrm{NMR}$ 光谱, 在 $\delta 153$ 处出 现的峰可归属于亚胺键中的碳, 这也表明我们成功合成 了 JUC-578(图 2b).

JUC-578 的结晶度可以通过 PXRD 测试进行确定. 如图 3a 所示, PXRD 在 $2 \theta=3.11^{\circ}, 5.28^{\circ}, 6.20^{\circ}$ 和 $8.00^{\circ}$ 处 的峰分别对应(100), (110), (200)和(120)晶面. 为了确定
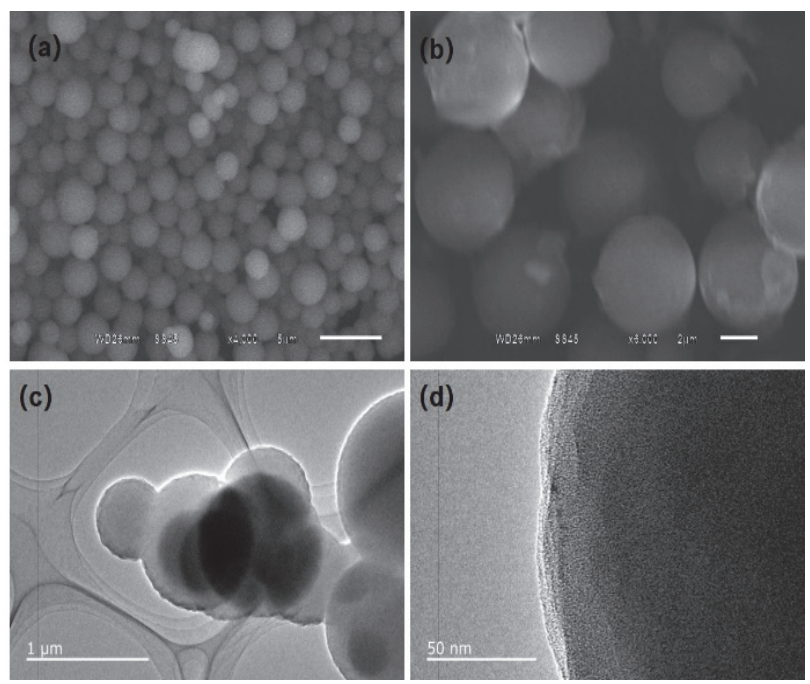

图 1 JUC-578 的 SEM 图(a,b)和 TEM 图(c,d)

Figure 1 (a, b) SEM and (c, d) TEM images of JUC-578 


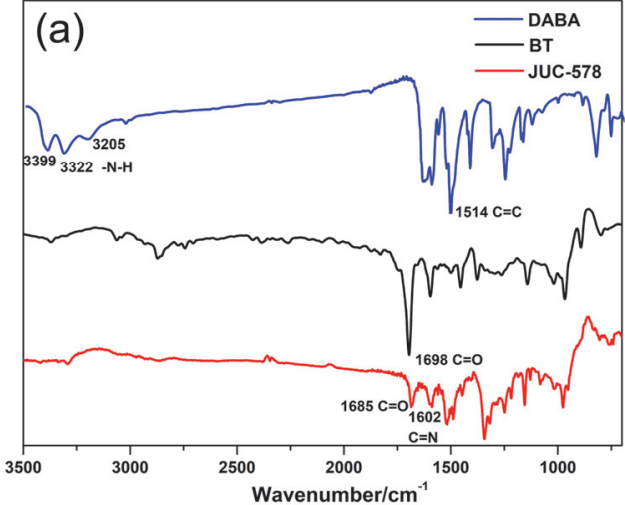

(b)

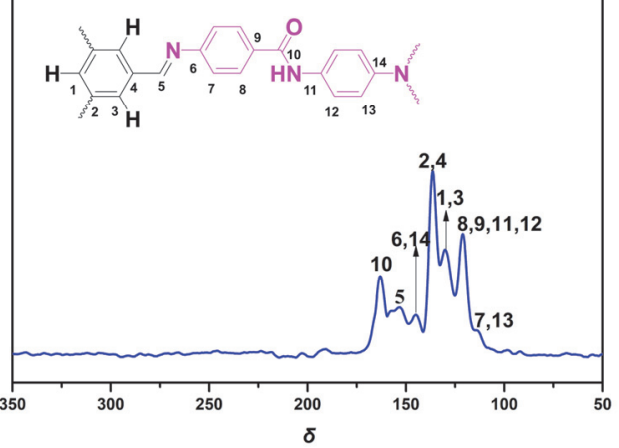

图 2 JUC-578 的(a) FT-IR 光谱(b)固态 ${ }^{13} \mathrm{C} \mathrm{NMR}$ 光谱

Figure 2 (a) FT-IR spectra and (b) solid-state ${ }^{13} \mathrm{C}$ NMR spectrum of JUC-578

JUC-578 的晶体结构, 我们使用 Materials Studio 软件包 构建了两种可能的二维模型: AA 堆积和 $\mathrm{AB}$ 堆积, 并模 拟了相应的粉末 $\mathrm{X}$ 射线衍射谱图. 其中 $\mathrm{AA}$ 堆积的晶胞 参数为 $a=b=3.351 \mathrm{~nm}, c=0.352 \mathrm{~nm}, \alpha=\beta=90^{\circ}, \gamma=$ $120^{\circ}, \mathrm{AB}$ 堆积的晶胞参数为 $a=b=3.351 \mathrm{~nm}, c=0.680$ $\mathrm{nm}, \alpha=\beta=90^{\circ}, \gamma=120^{\circ}$. 对比可以发现实验得到的 PXRD 图谱与 AA 堆积模型吻合良好. 我们将 Pawley 精 修得到的 PXRD 与实验所得进行拟合, 得到的 $R_{\omega \mathrm{p}}=$ $6.89 \%, R_{\mathrm{p}}=4.67 \%$, 表明二者吻合度较高. 基于这些结 果, 我们认为 JUC-578 是具有六边形介孔的 AA 堆积结 构.

为了研究 JUC-578 的热稳定性, 我们在氮气氛围下 进行了热重(TGA)测试. 结果显示, JUC-578 在高达 $430{ }^{\circ} \mathrm{C}$ 的温度下仍然稳定, 表明其具有很高的热稳定性 (图 3b), 而在 $430{ }^{\circ} \mathrm{C}$ 前出现的轻微的重量损失, 可能是 由于正己烷等溶剂蒸发导致的. JUC-578 的热重图谱从 $430{ }^{\circ} \mathrm{C}$ 后开始出现急剧的倾斜下降, 表明 JUC-578 的结 构在温度逐渐上升的情况下开始崩解, 直至 $800{ }^{\circ} \mathrm{C}$ 结 构被完全破坏. 我们通过在 $77 \mathrm{~K}$ 下进行 $\mathrm{N}_{2}$ 吸附分析来 确定孔隙率和比表面积. 通过计算 JUC-578 的 Brunauer-Emmett-Teller (BET)比表面积为 $46 \mathrm{~m}^{2} \cdot \mathrm{g}^{-1}$, 相 比类似报道的 BET 值 ${ }^{[22]}$, JUC-578 BET 低的原因可能是 移去溶剂后孔道收缩造成的 ${ }^{[23-25]}$. 利用非局部密度泛函
理论(NLDFT)计算了 JUC-578 的孔径分布, $2.8 \mathrm{~nm}$ 处显 示出较窄的强峰, 表明 JUC-578 具有一维介孔孔道.

(a)
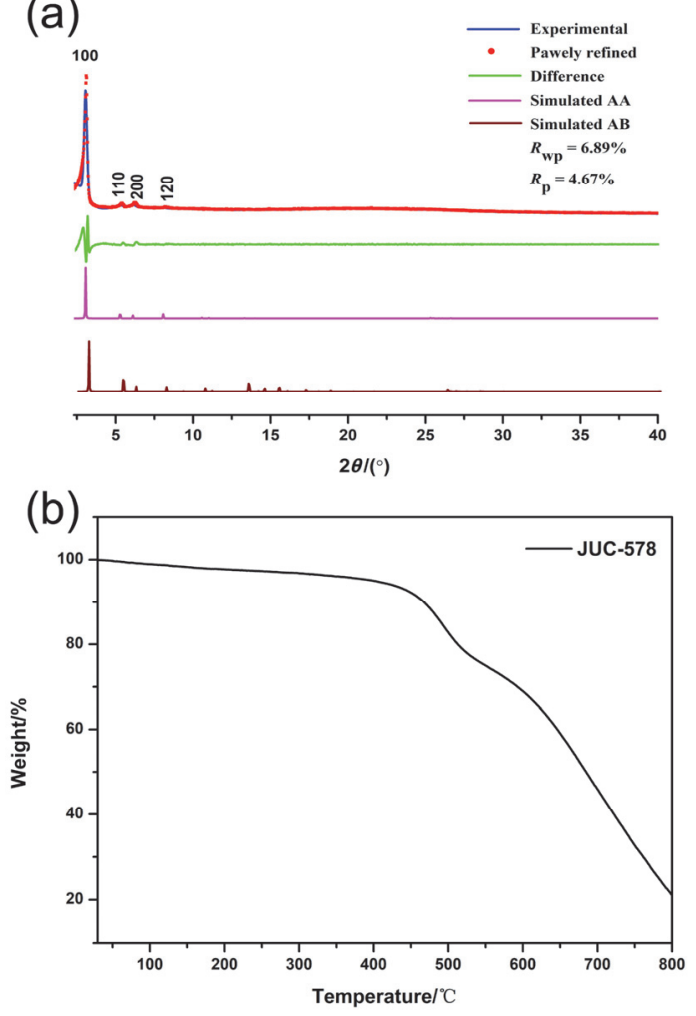

图 3 (a) JUC-578 的计算和实验 PXRD 和(b) TGA 曲线

Figure 3 (a) Calculated and experiment PXRD patterns, and (b) TGA curve of JUC-578

\section{2 染料的吸附}

为了研究对于染料的吸附性能, 我们挑选了具有不 同尺寸，带有不同电荷的四种染料进行了吸附实验，分 别是阳离子染料: 罗丹明 $6 \mathrm{G}$ (R6G), 亚甲基蓝 $\left(\mathrm{MB}^{+}\right)$阴 离子染料: 甲基橙 $\left(\mathrm{MO}^{-}\right)$, 橙黄 $\mathrm{G}\left(\mathrm{AO}^{-}\right)$. 取适量的 JUC-578 粉末分别浸泡在上述染料的水溶液中. 我们可 以通过直接观察染料溶液颜色的变化来判断吸附的程 度, 并且通过紫外可见吸收光谱对吸附过程进行定量的 检测. 结果显示, $\mathrm{MO}^{-}, \mathrm{AO}^{-}$溶液的颜色和浓度随时间几 乎保持不变, 而 $\mathrm{R} 6 \mathrm{G}, \mathrm{MB}^{+}$溶液的变化是非常明显的. 在吸附过程中, 二者的溶液颜色逐渐裉去, $110 \mathrm{~min}$ 后与 原溶液作对比可看到明显的颜色变浅(如图 4), 相反, 两 个阴离子染料的溶液颜色没有明显变化. 不同时间下测 定染料的吸附曲线也显示, 随着时间的推移， $\mathrm{R} 6 \mathrm{G}$ 和 $\mathrm{MB}^{+}$染料的浓度逐渐降低, 而 $\mathrm{MO}^{-}, \mathrm{AO}^{-}$染料的浓度不 发生变化. 上述现象表明, JUC-578 能够选择性地吸附 阳离子染料. 我们认为, JUC-578 之所以可以选择性地 吸附阳离子是因为骨架中暴露的具有孤电子对的氮活 性位点, 可作为电子给体与缺电子的阳离子染料发生相 互作用而具有选择性的吸附 ${ }^{[9,26]}$. 同时，骨架与染料之 间弱的相互作用(氢键、偶联作用、范德华力、 $\pi-\pi$ 相互 



图 4 JUC-578 对于(a) R6G, (c) $\mathrm{AO}^{-}$, (d) $\mathrm{MB}^{+}$和(f) $\mathrm{MO}^{-}$的染料溶液随时间变化的紫外-可见吸收光谱图以及(b) R6G 和(e) $\mathrm{MB}^{+}$的吸附效率曲线图 插入的图显示了染料溶液在吸附之前和吸附之后的颜色变化

Figure 4 Temporal evolution of the UV-Vis absorption spectra of aqueous solution containing (a) R6G, (c) $\mathrm{AO}^{-}$, (d) $\mathrm{MB}^{+}$and (f) $\mathrm{MO}^{-}$with JUC-578. The removal efficiency curves of (b) R6G and (e) $\mathrm{MB}^{+}$. Inserted graph shows the color of the dye solution before and after adsorption

作用)也对染料吸附做出了贡献 ${ }^{[27-28]}$. 通过 $\mathrm{COF}$ 粒子水 分散液的 zeta 电位的测试 $(-9.89 \mathrm{mV})$ ，也进一步证实了 较多的氮位点可以与阳离子染料之间存在强的静电相 互作用.

为了进一步验证该结论, 我们将 JUC-578 样品分别 浸入含有 $\mathrm{R} 6 \mathrm{G} / \mathrm{AO}^{-} 、 \mathrm{R} 6 \mathrm{G} / \mathrm{MO}^{-} 、 \mathrm{MB}^{+} / \mathrm{AO}^{-}$和 $\mathrm{MB}^{+} /$ $\mathrm{MO}^{-}$混合染料的水溶液中 (如图 5). 可以看出, 相对吸 收峰的强度变化非常明显, 其中与阳离子有关的峰的强 度急剧下降，而与其他染料有关的峰只有略微的偏移. 因此可以推断出, JUC-578 通过其一维的介孔孔道选择 性地从混合溶液中吸附阳离子染料, 而对阴离子只存在 非常少量的表面吸附 ${ }^{[6]}$. 为了进一步探究 JUC-578 对这 两种阳离子染料不同的吸附性能. 我们将 $\mathrm{R} 6 \mathrm{G}, \mathrm{MB}^{+}$配 制为相同浓度和体积比的二元溶液, 取适量的 JUC-578 加入到混合溶液中. 根据 Material Studio 软件计算, 染 料的分子大小为 $\mathrm{MB}^{+}<\mathrm{R} 6 \mathrm{G}$. 如图 5c 所示, R6G 在 526 $\mathrm{nm}$ 处的吸收峰强度有显著的降低, 而 $\mathrm{MB}^{+}$的吸收峰则 缓慢降低. 这可能是由于大尺寸染料更易与孔壁通过静 电相互作用结合, 从而占据了大部分的活性位点所导致 的. 与此同时, 绘制所得到的移除效率曲线也说明 JUC-578 具有的尺寸选择性.

为了实际应用吸附剂和目标染料来实现工业上的 循环利用, 我们用 $\mathrm{NaCl}$ 饱和的乙腈溶液进行了释放实 验. 如图 6a 所示, R6G 在 $30 \mathrm{~min}$ 内可被快速释放. 此
外, 在染料释放后进行了循环吸附实验. 从图 $6 \mathrm{~b}$ 中可以 看到，回收的 $\mathrm{COF}$ 可以循环使用多次. 同时, PXRD 测 试证明了该框架在染料吸附和释放的重复循环中仍保 持了母体 COF 的基本晶体结构. 因此, JUC-578 高的结 晶度、有序的孔道对于染料的吸附也是非常重要的因 素 ${ }^{[28-29]}$.

\section{3 结论}

综上所述, 我们利用 $\mathrm{DABA}$ 与 $\mathrm{BT}$ 的缩合反应成功 制备了酰胺功能化的二维 COF (JUC-578), 通过 PXRD、 SEM、TEM、 $\mathrm{N}_{2}$ 吸附、FT-IR 等表征手段证明了该 COF 具有高的结晶度, 均一规整的形貌, 以及开放的一维介 孔孔道. 通过将含氮功能基团引入骨架为 COF 提供了 更多的氮活性位点，利用 UV-Vis 检测了 JUC-578 对染 料的吸附过程, 由于骨架中富电子的氮可与缺电子的染 料发生静电作用以及骨架与染料其他弱的相互作用(氢 键、偶联作用、范德华力、 $\pi-\pi$ 相互作用), JUC-578 表现 出对阳离子染料 $\left(\mathrm{R} 6 \mathrm{G} 、 \mathrm{MB}^{+}\right)$的选择性吸附，而对于阴 离子染料 $\left(\mathrm{MO}^{-} 、 \mathrm{AO}^{-}\right)$只存在微量的表面吸附. 循环实 验表明 JUC-578 作为吸附剂可反复使用多次且不会破 坏其整体的结构. 这项工作为合理设计功能化 COF 用 于可持续生产以及环境保护方面提供了新的策略与方 向. 

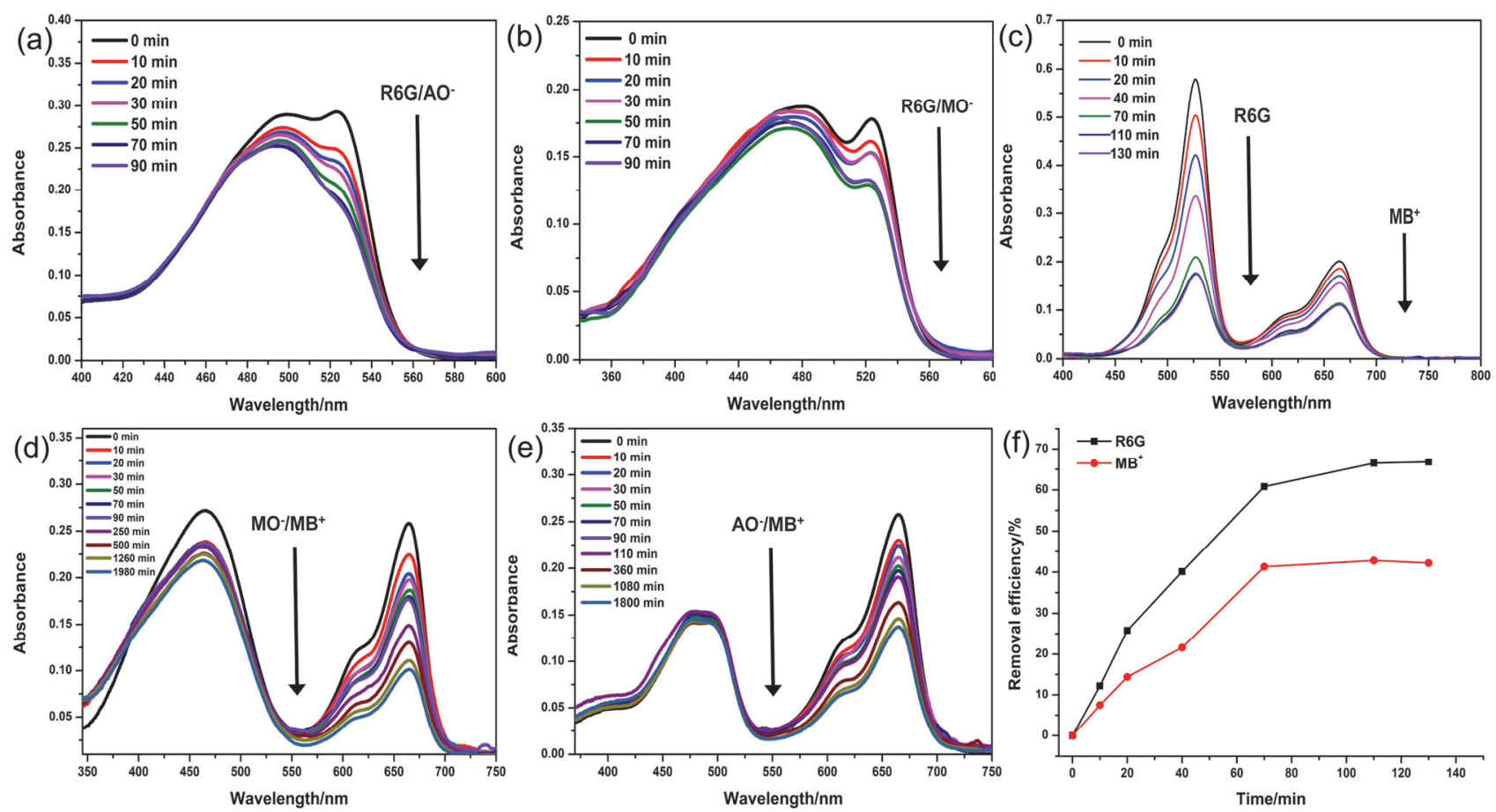

图 $5 \mathrm{JUC}-578$ 对于混合染料(a) R6G/AO , (b) R6G/MO ${ }^{-}$, (c) R6G/MB ${ }^{+}$, (d) $\mathrm{MB}^{+} / \mathrm{MO}^{-}$以及(e) $\mathrm{MB}^{+} / \mathrm{AO}^{-}$随时间变化的紫外-可见吸收光谱图. (f)混 合染料 $\mathrm{R} 6 \mathrm{G} / \mathrm{MB}^{+}$中不同染料的吸附效率图

Figure 5 Temporal evolution of the UV-Vis absorption spectra of mixed dyes solution containing (a) R6G/AO ${ }^{-}$, (b) R6G/MO ${ }^{-}$, (c) R6G/MB ${ }^{+}$, (d) MB ${ }^{+} /$ $\mathrm{MO}^{-}$, and (e) $\mathrm{MB}^{+} / \mathrm{AO}^{-}$with JUC-578. (f) The removal efficiency of different dyes in R6G/MB ${ }^{+}$solution
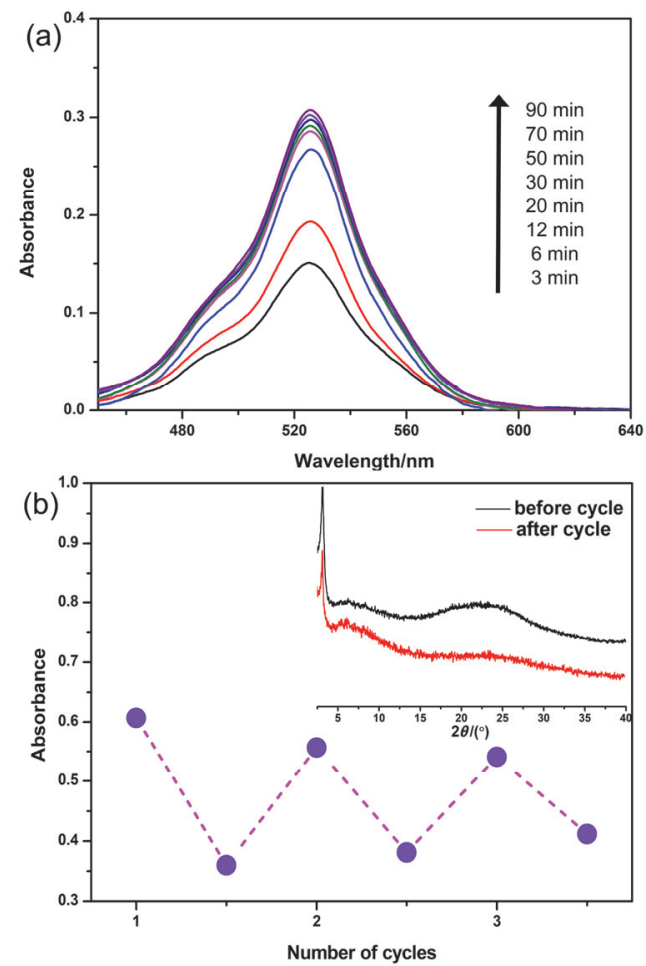

图 6 (a)吸附了 R6G 的 JUC-578 在氯化钠饱和的乙腈溶液中释放, (b) JUC-578 对于 R6G 的循环吸附图, 插图为循环之后与循环之前的 PXRD 对比

Figure 6 (a) R6G release experiment from the corresponding JUC-578 samples in $\mathrm{NaCl}$ saturated solution of $\mathrm{CH}_{3} \mathrm{CN}$. (b) Recurring multiple cycles of JUC-578 for R6G. Inserted graph shows the difference of PXRD before and after cycling

\section{4 实验部分}

\section{1 试剂与仪器}

均苯三甲醛(BT，纯度 $97 \%$, 吉林省研伸科技有限 公司); 4,4'-二氨基苯甲酰苯胺(DABA，纯度 $99 \%$ ，百灵 威科技有限公司), 无水 1,4-二氧六环(纯度 $99.5 \%$ ), 均 三甲苯(纯度 $98 \%$ ), 乙酸(纯度 $99.5 \%$, 阿拉丁试剂有限 公司), 正己烷(纯度 $97.5 \%$, 百灵威科技有限公司), 丙 酮(纯度 $97.5 \%$, 阿拉丁试剂有限公司), $N, N$-二甲基甲酰 胺(纯度 $\mathrm{AR}$, 西陇化工有限公司), 罗丹明 6G (R6G, 纯 度 $95 \%$ ，麦克林生化科技有限公司), 亚甲基蓝( $\mathrm{MB}$ ，纯 度 $97 \%$, 麦克林生化科技有限公司)，甲基橙( MO, 纯度 96\%，麦克林生化科技有限公司), 橙黄 G (AO, 纯度 $96 \%$ ，麦克林生化科技有限公司).

Empypean DY01610 粉末 X 射线衍射仪(PXRD, 荷 兰 PANalytical), JEM-2100F 型扫描电子显微镜(SEM, 日本电子株式会社), IRTracer-100 原位红外光谱仪(IR, 日本 Shimadzu 公司), DTG-60 型差热热重分析系统 (DTA-TG, 日本 Shimadzu 公司), UV-2450 分光光度计 (UV, 日本 Shimadzu 公司), Autosorb-iQ3 自动化气体吸 附分析仪(美国康塔)。

\section{2 酰胺功能化 COF 的合成}

我们将 DABA (34.09 $\mathrm{mg}, 0.15 \mathrm{mmol}$ )和 BT (16.21 $\mathrm{mg}, 0.10 \mathrm{~mol}$ )混合研磨，随后放入 Pyrex 管中，抽真空充氮气连续循环三次，除去管内空气并保持在纯氮气环 
境下，加入无水 1,4 -二氧六环 $(0.5 \mathrm{~mL})$ 、均三甲苯 $(0.5$ $\mathrm{mL})$ 和乙酸水溶液 $(3 \mathrm{~mol} / \mathrm{L}, 0.1 \mathrm{~mL})$. 接着将 Pyrex 管放 入 $77 \mathrm{~K}$ 液氮中快速冷冻, 抽真空至 $19.95 \mathrm{~Pa}$ 的内部压 力, 之后在 $\mathrm{CH}_{4}$ 与 $\mathrm{O}_{2}$ 混合气体火焰下将管密封, 管的长 度减少到约 $13 \mathrm{~cm}$, 将反应混合物放置于 $120{ }^{\circ} \mathrm{C}$ 烘箱中 加热 $3 \mathrm{~d}$, 过滤分离得到黄色沉淀物. 接着用丙酮 $(20$ $\mathrm{mL})$ 洗涤三次. 将产物浸入正己烷 $(20 \mathrm{~mL})$ 中 $8 \mathrm{~h}$, 在此 期间倾倒出活化溶剂, 并补充新鲜的正己烷溶剂. 最后 过滤得到固体, 放置于 $60{ }^{\circ} \mathrm{C}$ 烘箱中真空干燥 $2 \mathrm{~h}$, 得到 黄色粉末状的 JUC-578, 产率约为 $86 \%$.

\section{3 染料的吸附}

JUC-578 对水溶液中不同染料的吸附实验如下进 行: 将 $10 \mathrm{mg} \mathrm{JUC-578}$ 加到 $10 \mathrm{~mL}$ R6G、 $\mathrm{MB}^{+} 、 \mathrm{AO}^{-}$和 $\mathrm{MO}^{-}$的染料溶液 $(6 \mathrm{mg} / \mathrm{L})$ 中, 然后在室温下将悬浮液用 摇床分散混合. 静置后收集上清液, 并通过紫外可见分 光光度计对吸附不同时间的染料含量进行测定. 用紫外 可见分光光度法研究了吸附剂在二元混合溶液中的吸 附行为. 另外, 染料的释放实验在氯化钠饱和的乙腈溶 液中进行, 并循环多次用来测定吸附剂的可重复利用 性. 这些结果表明, JUC-578 可以用作从水溶液中选择 性吸收染料的吸附材料.

\section{References}

[1] An, Y.; Zheng, H.; Yu, Z.; Sun, Y.; Wang, Y.; Zhao, C.; Ding, W. J. Hazardous Mater. 2019, 381, 120971.

[2] Karimi-Maleh, H.; Shafieizadeh, M.; Taher, M. A.; Opoku, F.; Kiarii, E. M.; Govender, P. P.; Ranjbari, S.; Rezapour, M.; Orooji, Y. J. Mol. Liq. 2020, 298, 112040.

[3] Zheng, W.; Zhang J. H.; Jiang J. J.; Wang, H. P.; Wei, Z. W.; Zhu, X. J.; Pan, M.; Su, C.Y. J. Mater. Chem. A 2018, 6, 17698.

[4] Liu, X. D.; Tian, J. F.; Li, Y. Y.; Sun, N. F.; Mi, S.; Xie, Y.; Chen, Z. Y. J. Hazard. Mater. 2019, 373, 397.

[5] Im, K.; Nguyen, D. N.; Kim, S.; Kong, H. J.; Kim, Y.; Park, C. S.; Kwon, O. S.; Yoon, H. ACS Appl. Mater. Interfaces 2017, 9, 10768.

[6] Chen, F.; Zhao, E.; Kim, T.; Wang, J.; Hableel, G.; Reardon, P. J. T.;
Ananthakrishna, S. J.; Wang, T.; Arconada-Alvarez, S.; Knowles, J. C.; Jokerst, J. V. ACS Appl. Mater. Interfaces 2017, 9, 15566.

[7] Shen, Y.; Fan, C. C.; Wei, Y. Z.; Du, J.; Zhu, H. B.; Zhao, Y. Dalton Trans. 2016, 45, 10909 .

[8] Wang, D.; Zhang, J.; Li, G.; Yuan, J.; Li, J.; Huo, Q.; Liu, Y. ACS Appl. Mater. Interfaces 2018, 10, 31233.

[9] Wang, Z.; Zhu, C. Y.; Zhao, H. S.; Yin, S. Y.; Wang, S. J.; Zhang, J. H.; Jiang, J. J.; Pan, M.; Su, C. Y. J. Mater. Chem. A. 2019, 7, 4751.

[10] Zhu, X.; An, S. H.; Liu, Y.; Hu, Jun.; Liu, H. L.; Tian, C. C.; Dai, S.; Yang, X. J.; Wang, H. L.; Abney, C. W. AIChE. J. 2017, 63, 3470.

[11] Côté, A. P.; Benin, A. I.; Ockwig, N. W.; O'Keeffe, M.; Matzger, A. J.; Yaghi, O. M. Science 2005, 310, 1166.

[12] Furukawa, H.; Yaghi, O. M. J. Am. Chem. Soc. 2009, 131, 8875.

[13] Fu, J. R.; Beng, T. Acta Chim. Sinica 2020, 78, 805. (付静茹, 贲腾, 化学学报, 2020, 78, 805.)

[14] Li, H.; Pan, Q. Y.; Ma, Y. C.; Guan, X. Y.; Xue, M.; Fang, Q. R.; Yan, Y. S.; Valtchev, V.; Qiu, S. L. J. Am. Chem. Soc. 2016, 138, 14783.

[15] Yang, L.; Wei, D. C. Chin. Chem. Lett. 2016, 27, 1395.

[16] Ma, L.; Wang, S.; Feng, X.; Wang, B. Chin. Chem. Lett. 2016, 27, 1383.

[17] Dalapati, S.; Jin, S. B.; Gao, J.; Xu, Y. H.; Nagai, A.; Jiang, D. L. J. Am. Chem. Soc. 2013, 135, 17310.

[18] Wang, Z. T.; Li, H.; Yan, S. C.; Fang, Q. R. Acta Chim. Sinica 2020, 78, 63. (王志涛, 李辉, 颜士臣, 方千荣, 化学学报, 2020, 78, 63.)

[19] Xu, T.; An, S. H.; Peng, C. J.; Hu, J.; Liu, H. L. Ind. Eng. Chem. Res. 2020, 59, 8315.

[20] Wang, T.; Kailasam, K. K.; Xiao, P.; Chen, G. S.; Chen, L. Q.; Wang, L. H.; Li, J. L.; Ju, J. J. Micropor. Mesopor. Mat. 2014, 187, 63.

[21] Luo, D.; Wang, X. Z.; Zhang, Z. R.; Gao, D. N.; Liu, Z. J.; Chen, J. B. Int. J. Hydrogen Energ. 2020, 45, 30375.

[22] Qian, H. L.; Meng, F. L.; Yang, C. X.; Yan, X. P. Angew. Chem. Int. Ed. 2020, 59, 17607.

[23] Li, D. H.; Li, C. Y.; Zhang, L. J.; Li, H.; Zhu, L. K.; Yang, D. J.; Fang, Q.R.; Qiu, S. L.; Yao, X. D. J. Am. Chem. Soc. 2020, 142, 8104 .

[24] Ma, Y. X.; Li, Z. J.; Wei, L.; Ding, S. Y.; Zhang, Y. B.; Wang, W. J. Am. Chem. Soc. 2017, 139, 4995.

[25] Wang, Y. J.; Liu, Y. Z.; Li, H.; Guan, X. Y.; Xue, M.; Yan, Y. S.; Valtchev, V.; Qiu, S. L. Fang, Q. R. J. Am. Chem. Soc. 2020, 142, 3736.

[26] Wang, Z.; Zhu, C. Y.; Mo, J. T.; Fu, P. Y.; Zhao, Y. W.; Yin, S. Y.; Jiang, J. J.; Pan, M.; Su, C. Y. Angew. Chem. Int. Ed. 2019, 58, 9752.

[27] Liao, Q. B.; Ke, C.; Huang, X. Zhang, G. Y.; Zhang, Q.; Zhang, Z. W.; Zhang, Y. Y.; Liu, Y. Z.; Ning, F. Y.; Xi, K. J. Mater. Chem. A 2019, 7, 18959.

[28] Firoozi, M.; Rafiee, Z.; Dashtian, K. ACS Omega 2020, 5, 9420.

[29] Li, H.; An, N. H.; Liu, G.; Li, J. L.; Liu, N.; Jia, M. J.; Zhang, W. X.; Yuan, X. L. J. Colloid Interf. Sci. 2016, 466, 343. 